\title{
New Properties for The Ramanujan'S Continued Fraction of Order 12
}

\author{
Chandrashekar Adiga*, M. S. Surekha, A. Vanitha \\ Department of Studies in Mathematics, University of Mysore, Manasagangotri, Mysore 570 006, INDIA \\ *Corresponding author: c_adiga@hotmail.com
}

Received May 07, 2014; Revised June 24, 2014; Accepted July 03, 2014

\begin{abstract}
In this paper, we derive new identities involving a continued fraction of Ramanujan of order twelve that are similar to those of the Ramanujan-Göllnitz-Gordon continued fraction.
\end{abstract}

Keywords: continued fraction, power series expansion

Cite This Article: Chandrashekar Adiga, M. S. Surekha, and A. Vanitha, "New Properties for The Ramanujan'S Continued Fraction of Order 12." Turkish Journal of Analysis and Number Theory, vol. 2, no. 3 (2014): 90-95. doi: 10.12691/tjant-2-3-7.

\section{Introduction}

The celebrated Ramanujan-Göllnitz-Gordon continued fraction is defined by

$$
H(q):=\frac{q^{1 / 2}}{1+q}+\frac{q^{2}}{1+q^{3}}+\frac{q^{4}}{1+q^{5}}+\frac{q^{6}}{1+q^{7}}+\ldots .|q|<1 .
$$

On page 229 of his second notebook [10], Ramanujan recorded a product representation of $H(q)$, namely

$$
H(q)=q^{1 / 2} \frac{\left(q ; q^{8}\right)_{\infty}\left(q^{7} ; q^{8}\right)_{\infty}}{\left(q^{3} ; q^{8}\right)_{\infty}\left(q^{5} ; q^{8}\right)_{\infty}}
$$

Throughout this paper, we assume that $|q|<1$ and use the standard notation

$$
(a ; q)_{n}=\prod_{j=0}^{n-1}\left(1-a q^{j}\right) \text { and }(a ; q)_{\infty}=\prod_{j=0}^{\infty}\left(1-a q^{j}\right) .
$$

For convenience, we often write $\left(a_{1}, a_{2}, \ldots a_{k} ; q\right)_{\infty}$ for $\left(a_{1} ; q\right)_{\infty}, \ldots\left(a_{k} ; q\right)_{\infty}$ in the sequel.

Ramanujan's general theta function is defined by

$$
f(a, b):=\sum_{n=-\infty}^{\infty} a^{n(n+1) / 2} b^{n(n-1) / 2},|a b|<1 .
$$

The Jacobi triple product identity [1] in Ramanujan's notation is

$$
f(a, b)=(-a ; a b)_{\infty}(-b ; a b)_{\infty}(a b ; a b)_{\infty} .
$$

Jacobi's triple product identity is a special case of ${ }_{1} \psi_{1}$ summation formula [1] due to Ramanujan. S. Bhargava et al. [6] made use of Ramanujan's ${ }_{1} \psi_{1}$ summation formula to prove a convolution identity for certain coefficientsgenerated by the quotient of two infinite products. As special cases of this identity, they deduced several results including, for example, the convolution identities given earlier by Kung - Wei Yang [15]. In [6], Bhargava et al. raised an interesting question concerning the derivability of convolution identity as a conseuence of ${ }_{2} \phi_{1}$ summation formula instead of the ${ }_{1} \psi_{1}$ and ${ }_{2} \psi_{2}$ sums. This question was completely answered by $\mathrm{H}$. M. Srivastava[12].

The four important special cases of $f(a, b)[1]$ are

$$
\begin{gathered}
\phi(q):=f(q, q)=\sum_{n=-\infty}^{\infty} q^{n^{2}}=\frac{\left(q^{2} ; q^{2}\right)_{\infty}^{5}}{(q ; q)_{\infty}^{2}\left(q^{4} ; q^{4}\right)_{\infty}^{2}}, \\
\phi(-q):=f(-q,-q)=\sum_{n=-\infty}^{\infty}(-q)^{n^{2}}=\frac{(q ; q)_{\infty}^{2}}{\left(q^{2} ; q^{2}\right)_{\infty}}, \\
\psi(q):=f\left(q, q^{3}\right)=\sum_{n=0}^{\infty} q^{n(n+1) / 2}=\frac{\left(q^{2} ; q^{2}\right)_{\infty}^{2}}{(q ; q)_{\infty}}
\end{gathered}
$$

and

$$
f(-q):=f\left(-q,-q^{2}\right)=\sum_{n=-\infty}^{\infty}(-1)^{n} q^{n(3 n-1) / 2}=(q ; q)_{\infty} .
$$

Also, after Ramanujan, define

$$
\chi(q):=\left(-q ; q^{2}\right)_{\infty}=\frac{\left(q^{2} ; q^{2}\right)_{\infty}^{2}}{(q ; q)_{\infty}\left(q^{4} ; q^{4}\right)_{\infty}} .
$$

Ramanujan recorded many identities involving $f(a, b), \phi(q), \psi(q)$ and $\chi(q)$.

The famous Göllnitz-Gordon functions $S(q)$ and $T(q)$ are defined by

$S(q):=\sum_{n=0}^{\infty} \frac{\left(-q ; q^{2}\right)_{n}}{\left(q^{2} ; q^{2}\right)_{n}} q^{n^{2}}=\frac{1}{\left(q ; q^{8}\right)_{\infty}\left(q^{4} ; q^{8}\right)_{\infty}\left(q^{7} ; q^{8}\right)_{\infty}}(9)$ 
and

$$
\begin{aligned}
& T(q):=\sum_{n=0}^{\infty} \frac{\left(-q ; q^{2}\right)_{n}}{\left(q^{2} ; q^{2}\right)_{n}} q^{n^{2}+2 n} \\
& =\frac{1}{\left(q^{3} ; q^{8}\right)_{\infty}\left(q^{4} ; q^{8}\right)_{\infty}\left(q^{5} ; q^{8}\right)_{\infty}},
\end{aligned}
$$

where the two equalities on the righthand sides of (9) and (10) are the celebrated Göllinitz-Gordon identities [7,8].

We note that

$$
H(q)=q^{1 / 2} \frac{T(q)}{S(q)} .
$$

Without any knowledge of Ramanujan's work, $\mathrm{H}$. Göllnitz [7] and B. Gordon [8] rediscovered and proved (2) independently. Later G. E. Andrew [3] proved (2) as a corollary of a more general result. Moreover, Ramanujan established two further identities for $H(q),[5,10]$, namely,

$$
\frac{1}{H(q)}-H(q)=\frac{\left(-q^{2} ; q^{4}\right)_{\infty}^{2}\left(q^{4} ; q^{4}\right)_{\infty}\left(q^{4} ; q^{8}\right)_{\infty}}{q^{1 / 2}\left(q^{8} ; q^{8}\right)_{\infty}}
$$

and

$$
\frac{1}{H(q)}+H(q)=\frac{\left(-q ; q^{2}\right)_{\infty}^{2}\left(q^{2} ; q^{2}\right)_{\infty}\left(q^{4} ; q^{8}\right)_{\infty}}{q^{1 / 2}\left(q^{8} ; q^{8}\right)_{\infty}} .
$$

In [6], Boonrod Yuttanan found that

$$
\frac{1}{H(q)}+2-H(q)=\frac{\phi\left(q^{1 / 2}\right)}{q^{1 / 2} \psi\left(q^{4}\right)}
$$

and

$$
\frac{1}{H(q)}-2-H(q)=\frac{\phi\left(-q^{1 / 2}\right)}{q^{1 / 2} \psi\left(q^{4}\right)} .
$$

The following continued fraction (15) was established by M. S. M. Naika et al. [9] as a special case of a fascinating continued fraction identity recorded by Ramanujan in his Second Notebook [10]:

$$
\begin{gathered}
U(q):=\frac{q(1-q)}{\left(1-q^{3}\right)}+\frac{q^{3}\left(1-q^{2}\right)\left(1-q^{4}\right)}{\left(1-q^{3}\right)\left(1+q^{6}\right)} \\
+\ldots+\frac{q^{3}\left(1-q^{6 n-4}\right)\left(1-q^{6 n-2}\right)}{\left(1-q^{3}\right)\left(1+q^{6 n}\right)}+\ldots
\end{gathered}
$$

Now, we have

$$
U(q)=\frac{q f\left(-q,-q^{11}\right)}{f\left(-q^{5},-q^{7}\right)}=\frac{q N(q)}{M(q)},
$$

where

$$
M(q)=\frac{f\left(-q^{5},-q^{7}\right)}{f_{4}} \text { and } N(q)=\frac{f\left(-q,-q^{11}\right)}{f_{4}} .
$$

We define

$$
U^{*}(q):=\frac{N(q)}{M(q)} \text { and } V^{*}(q):=\frac{1}{U^{*}(q)} .
$$

Motivated by the identities (11)-(14) involving the Ramanujan-Göllnitz-Gordon continued fraction, in this paper, we establish several new identities and properties of $U(q)$.

\section{Preliminaries}

The following Lemmas are useful to prove our main results.

Lemma 2.1. [9]. We have

$$
\begin{aligned}
& \phi(q)+\phi\left(q^{3}\right)=2 \chi(q) f\left(-q^{5},-q^{7}\right), \\
& \phi(q)-\phi\left(q^{3}\right)=2 q \chi(q) f\left(-q,-q^{11}\right) .
\end{aligned}
$$

Lemma 2.2. [2]. We have

$$
\frac{\phi^{2}(q)}{\phi^{2}\left(q^{3}\right)}-1=\frac{4 q \psi(q) \psi^{3}\left(q^{6}\right)}{\psi\left(q^{2}\right) \psi^{3}\left(q^{3}\right)} .
$$

Lemma 2.3. [1]. We have

$$
\begin{gathered}
f(a, b)=f\left(a^{3} b, a b^{3}\right)+a f\left(b / a, a^{5} b^{3}\right), \\
\phi(q) \psi\left(q^{2}\right)=\psi^{2}(q), \\
f(q) \chi(q)=\phi(q), \\
f(a, b) f(-a,-b)=f\left(-a^{2},-b^{2}\right) \phi(-a b) .
\end{gathered}
$$

Lemma 2.4. [4]. We have

$$
\phi^{2}(q)+\phi^{2}\left(q^{3}\right)=\frac{2 \phi\left(-q^{2}\right) \phi\left(-q^{3}\right) \phi\left(-q^{6}\right)}{\phi(-q)} .
$$

Lemma 2.5. [9]. We have

$$
\frac{\phi(q)}{\phi\left(q^{3}\right)}=\frac{1+U(q)}{1-U(q)} .
$$

Lemma 2.6. [11]. We have

$$
\frac{\phi^{2}(q)}{\phi^{2}\left(q^{3}\right)}=1-\frac{4 U(-q)}{1+U^{2}(-q)} \text {. }
$$

Lemma 2.7. [1]. We have

$$
f_{4}^{2} \chi^{2}(q)=\psi^{2}(q)
$$

and

$$
\phi(q) \phi(-q)=\phi^{2}\left(-q^{2}\right),
$$

where $f_{n}:=f\left(-q^{n}\right)=\left(q^{n} ; q^{n}\right)_{\infty}$.

Lemma 2.8. [12]. We have

$$
\phi^{2}(q)+3 \phi^{2}\left(q^{3}\right)=4 \frac{\phi\left(q^{3}\right) \psi^{3}(q)}{\phi(q) \psi\left(q^{3}\right)} .
$$

Lemma 2.9. [1]. Let $U_{n}=a^{n(n+1) / 2} b^{n(n-1) / 2}$ and $V_{n}=a^{n(n-1) / 2} b^{n(n+1) / 2}$ for each integer $n$. Then

$$
f\left(U_{1}, V_{1}\right)=\sum_{r=0}^{k-1} U_{r} f\left(\frac{U_{k+r}}{U_{r}}, \frac{V_{k-r}}{U_{r}}\right),
$$


for every positive integer $k$.

Lemma 2.10. [1]. We have

$$
f(a, b)=f(b, a)
$$

and if $n$ is an integer, then

$$
f(a, b)=a^{n(n+1) / 2} b^{n(n-1) / 2} f\left(a(a b)^{n}, b(a b)^{-n}\right) .
$$

\section{Main Results}

In this section, we establish some new identities involving the continued fraction $U(q)$, which resemble (11)-(14) and Theorem 3.1 of [6].

Theorem 3.1. We have

$$
\begin{gathered}
\frac{1}{U(q)}-U(q)=\frac{\psi(q) \psi\left(q^{3}\right)}{q \psi^{2}\left(q^{6}\right)}, \\
\frac{1}{U(q)}+U(q)=\frac{\chi^{3}\left(-q^{3}\right) \chi^{3}\left(-q^{6}\right)}{q \chi(-q) \chi\left(-q^{2}\right)}, \\
\frac{1}{\sqrt{U(q)}}+\sqrt{U(q)}=\frac{\phi(q) \sqrt{\psi\left(q^{2}\right) \psi^{3}\left(q^{3}\right)}}{q^{1 / 2} \phi\left(q^{3}\right) \sqrt{\psi(q) \psi^{3}\left(q^{6}\right)}}, \\
\frac{1}{\sqrt{U(q)}}-\sqrt{U(q)}=\frac{f\left(-q, q^{2}\right) \chi(q) \sqrt{\psi\left(q^{2}\right) \psi^{3}\left(q^{3}\right)}}{q^{1 / 2} \phi\left(q^{3}\right) \sqrt{\psi(q) \psi^{3}\left(q^{6}\right)}} .
\end{gathered}
$$

Proof. By Lemma 2.1, we have

$$
\frac{1}{U(q)}-U(q)=\frac{\phi(q)+\phi\left(q^{3}\right)}{\phi(q)-\phi\left(q^{3}\right)}-\frac{\phi(q)-\phi\left(q^{3}\right)}{\phi(q)+\phi\left(q^{3}\right)} .
$$

On simplification of (37), we obtain

$$
\frac{1}{U(q)}-U(q)=\frac{4 \phi(q) \phi\left(q^{3}\right)}{\phi^{2}\left(q^{3}\right)\left(\frac{\phi^{2}(q)}{\phi^{2}\left(q^{3}\right)}-1\right)} .
$$

Employing (20) in (38), we can deduce that

$$
\frac{1}{U(q)}-U(q)=\frac{\phi(q) \psi\left(q^{2}\right) \psi^{3}\left(q^{3}\right)}{q \phi\left(q^{3}\right) \psi(q) \psi^{3}\left(q^{6}\right)} .
$$

Using (22) in (39), we establish that

$$
\frac{1}{U(q)}-U(q)=\frac{\psi(q) \psi\left(q^{3}\right)}{q \psi^{2}\left(q^{6}\right)} .
$$

By Lemma 2.1, we have

$$
\frac{1}{U(q)}+U(q)=\frac{\phi(q)+\phi\left(q^{3}\right)}{\phi(q)-\phi\left(q^{3}\right)}+\frac{\phi(q)-\phi\left(q^{3}\right)}{\phi(q)+\phi\left(q^{3}\right)} .
$$

On simplification of (40), we obtain

$$
\frac{1}{U(q)}+U(q)=\frac{2\left(\phi^{2}(q)+\phi^{2}\left(q^{3}\right)\right)}{\phi^{2}\left(q^{3}\right)\left(\frac{\phi^{2}(q)}{\phi^{2}\left(q^{3}\right)}-1\right)} .
$$

Employing (20) and (25) in (41), we get

$$
\frac{1}{U(q)}+U(q)=\frac{\phi\left(-q^{2}\right) \phi\left(-q^{3}\right) \phi\left(-q^{6}\right) \psi\left(q^{2}\right) \psi^{3}\left(q^{3}\right)}{q \phi(-q) \phi^{2}\left(q^{3}\right) \psi(q) \psi^{3}\left(q^{6}\right)} .
$$

Using (22) in (42), we deduce that

$$
\frac{1}{U(q)}+U(q)=\frac{\phi\left(-q^{2}\right) \phi\left(-q^{3}\right) \phi\left(-q^{6}\right) \psi\left(q^{2}\right)}{q \phi(-q) \psi\left(q^{6}\right) \psi(q) \psi\left(q^{3}\right)} .
$$

It is easy to check that

$$
\begin{gathered}
\frac{\phi(-q)}{\psi(q)}=\chi^{3}(-q), \\
\frac{\phi\left(-q^{2}\right)}{\phi(-q)}=\frac{\chi(q)}{\chi(-q)}, \\
\frac{\psi\left(q^{2}\right)}{\psi(q)}=\frac{1}{\chi(q) \chi\left(-q^{2}\right)} .
\end{gathered}
$$

Employing (44), (45) and (46), in (43), we obtain (34). Using the definition of $U(q)$, we have

$$
\frac{1}{\sqrt{U(q)}}+\sqrt{U(q)}=\frac{f\left(-q^{5},-q^{7}\right)+q f\left(-q,-q^{11}\right)}{\sqrt{q f\left(-q,-q^{11}\right) f\left(-q^{5},-q^{7}\right)}} .
$$

Putting $a=q$ and $b=-q^{2}$ in (21), we obtain

$$
f\left(-q^{5},-q^{7}\right)+q f\left(-q,-q^{11}\right)=f\left(q,-q^{2}\right) .
$$

Employing (48) and Lemma 2.1. in (47), we get

$$
\begin{aligned}
& \frac{1}{\sqrt{U(q)}}+\sqrt{U(q)}=\frac{2 f(q) \chi(q)}{\sqrt{\left(\phi^{2}(q)-\phi^{2}\left(q^{3}\right)\right)}} \\
& =\frac{2 f(q) \chi(q)}{\phi\left(q^{3}\right) \sqrt{\left(\frac{\phi^{2}(q)}{\phi^{2}\left(q^{3}\right)}-1\right)}} .
\end{aligned}
$$

Employing (20) and (23) in (49), we obtain (35).

The proof of (36) is similar to the proof of (35).

Remark 3.2. Squaring (35) and (36) and after some simplifications, we obtain

$$
\begin{aligned}
& \frac{1}{U(q)}+2+U(q)=\frac{\psi(q) \phi(q)}{q \psi\left(q^{3}\right) \psi\left(q^{6}\right)} \\
& \frac{1}{U(q)}-2+U(q)=\frac{\psi\left(q^{2}\right) \psi^{3}\left(q^{3}\right)}{q \psi(q) \psi^{3}\left(q^{6}\right)} .
\end{aligned}
$$

The results in the following corollary are simply the squares (or product) of the identities (33) and (34).

Corollary 3.3. We have

$$
\begin{gathered}
\frac{1}{U^{2}(q)}-2+U^{2}(q)=\frac{\psi^{2}(q) \psi^{2}\left(q^{3}\right)}{q^{2} \psi^{4}\left(q^{6}\right)}, \\
\frac{1}{U^{2}(q)}+2+U^{2}(q)=\frac{\phi^{3}\left(-q^{3}\right)}{q^{2} \chi^{2}(-q) \chi^{2}\left(-q^{2}\right) \psi^{3}\left(q^{6}\right)}, \\
\frac{1}{q^{2} U^{2}(q)}-q^{2} U^{2}(q)=\frac{\phi^{3}\left(-q^{6}\right) \phi\left(-q^{2}\right) \psi\left(q^{2}\right)}{\phi(-q) \psi^{2}\left(q^{3}\right) \psi^{2}\left(q^{6}\right)} .
\end{gathered}
$$




$$
x\left(2 y-1-y^{2}-x y\right)-y=0,
$$

where $x=U(q)$ and $y=U(-q)$.

Proof. From Lemma 2.1, we have

$$
\begin{aligned}
& U(q)-U^{2}(q) \\
& =\frac{\phi(q)-\phi\left(q^{3}\right)}{\phi(q)+\phi\left(q^{3}\right)}-\frac{\left(\phi(q)-\phi\left(q^{3}\right)\right)^{2}}{\left(\phi(q)+\phi\left(q^{3}\right)\right)^{2}} \\
& =\frac{-2\left(1-\frac{\phi(q)}{\phi\left(q^{3}\right)}\right)}{1+\frac{\phi^{2}(q)}{\phi^{2}\left(q^{3}\right)}+2 \frac{\phi(q)}{\phi\left(q^{3}\right)}} .
\end{aligned}
$$

Using (26) and Lemma 2.6 in (54), we obtain (53).

Theorem 3.5. For $|q|<1$,

$$
\begin{gathered}
M^{2}(q)+q^{2} N^{2}(q)=\frac{\phi\left(-q^{2}\right) \phi\left(-q^{3}\right) \phi\left(-q^{6}\right)}{\phi(-q) \psi^{2}(q)}, \\
M^{2}(q)-q^{2} N^{2}(q)=\frac{\phi(q) \phi\left(q^{3}\right)}{\psi^{2}(q)},
\end{gathered}
$$

or equivalently

$$
\begin{aligned}
& V^{*}(q)=\frac{\left(q^{3} ; q^{3}\right)_{\infty}\left(q^{4} ; q^{4}\right)_{\infty}}{2(q ; q)_{\infty}\left(q^{6} ; q^{6}\right)_{\infty}\left(q^{12} ; q^{12}\right)_{\infty}^{2}} \\
& \sum_{m, n=-\infty}^{\infty}\left((-1)^{m+n} q^{6 m^{2}+3 n^{2}}+(-1)^{m} q^{2 m^{2}+3 n^{2}}\right), \\
& U^{*}(q)=\frac{\left(q^{3} ; q^{3}\right)_{\infty}\left(q^{4} ; q^{4}\right)_{\infty}}{2 q^{2}(q ; q)_{\infty}\left(q^{6} ; q^{6}\right)_{\infty}\left(q^{12} ; q^{12}\right)_{\infty}^{2}} \\
& \sum_{m, n=-\infty}^{\infty}\left((-1)^{m+n} q^{6 m^{2}+3 n^{2}}-(-1)^{m} q^{2 m^{2}+3 n^{2}}\right) .
\end{aligned}
$$

Proof. By the definitions of $M(q)$ and $N(q)$, we have

$$
\begin{aligned}
& M^{2}(q)+q^{2} N^{2}(q) \\
& =\frac{1}{f_{4}^{2}}\left\{f^{2}\left(-q^{5},-q^{7}\right)+q^{2} f^{2}\left(-q,-q^{11}\right)\right\} .
\end{aligned}
$$

Employing (18) and (19) in (59) and after some simplifications, we deduce

$$
M^{2}(q)+q^{2} N^{2}(q)=\frac{\left(\phi^{2}(q)+\phi^{2}\left(q^{3}\right)\right)}{2 f_{4}^{2} \chi^{2}(q)} .
$$

Employing (25) and (28) in (60), we obtain (55).

The proof of (56) is similar to the proof of (55).

Dividing both sides of (55) and (56), by $M(q) N(q)$ and then employing (18), (19) and (20) in resulting identities, we arrive at

$$
\begin{gathered}
V^{*}(q)+q^{2} U^{*}(q) \\
=\frac{\phi\left(-q^{2}\right) \phi\left(-q^{3}\right) \phi\left(-q^{6}\right) \chi^{2}(q) \psi\left(q^{2}\right) \psi^{3}\left(q^{3}\right) f_{4}^{2}}{\phi^{2}\left(q^{3}\right) \phi(-q) \psi^{3}(q) \psi^{3}\left(q^{6}\right)}, \\
V^{*}(q)-q^{2} U^{*}(q)=\frac{\phi(q) \phi\left(q^{3}\right) \chi^{2}(q) \psi\left(q^{2}\right) \psi^{3}\left(q^{3}\right) f_{4}^{2}}{\phi^{2}\left(q^{3}\right) \psi^{3}(q) \psi^{3}\left(q^{6}\right)} .
\end{gathered}
$$

Next, by adding (61) to (62), we obtain

$V^{*}(q)=\frac{\chi^{2}(q) \psi\left(q^{2}\right) \psi^{3}\left(q^{3}\right) f_{4}^{2}}{2 \phi^{2}\left(q^{3}\right) \psi^{3}(q) \psi^{3}\left(q^{6}\right) \phi(-q)}$

$\left\{\phi\left(-q^{2}\right) \phi\left(-q^{3}\right) \phi\left(-q^{6}\right)+\phi(-q) \phi(q) \phi\left(q^{3}\right)\right\}$.

Subtracting (62) from (61), we get

$$
\begin{aligned}
& U^{*}(q)=\frac{\chi^{2}(q) \psi\left(q^{2}\right) \psi^{3}\left(q^{3}\right) f_{4}^{2}}{2 q^{2} \phi(-q) \phi^{2}\left(q^{3}\right) \psi^{3}(q) \psi^{3}\left(q^{6}\right)} \\
& \left\{\phi\left(-q^{2}\right) \phi\left(-q^{3}\right) \phi\left(-q^{6}\right)-\phi(-q) \phi(q) \phi\left(q^{3}\right)\right\} .
\end{aligned}
$$

Employing (29), (5), (6), (7), (8) and definition of $\chi(q)$ in above two identities, we get (57) and (58). The arguments above can be easily reversed to show that (57) and (58) imply (55) and (56).

Theorem 3.6. We have

$$
\begin{gathered}
\frac{1+U^{3}(q)}{U(q)-U^{2}(q)}=\frac{\psi^{2}(q) \psi\left(q^{2}\right)}{q \psi\left(q^{6}\right) \psi^{2}\left(q^{3}\right)} \\
\frac{1+U^{3}(q)}{(1-U(q))^{3}}=\frac{\psi^{3}(q)}{\phi^{2}\left(q^{3}\right) \psi\left(q^{3}\right)} \\
\frac{1-U^{4}(q)}{(1-U(q))^{4}}=\frac{\phi^{2}(q) \phi\left(-q^{2}\right) \phi\left(-q^{3}\right) \phi\left(-q^{6}\right)}{\phi^{2}\left(q^{3}\right)} .
\end{gathered}
$$

Proof. By Lemma 2.1, we have

$$
\frac{1}{U^{2}(q)}+U(q)=\frac{\left(\phi(q)+\phi\left(q^{3}\right)\right)^{2}}{\left(\phi(q)-\phi\left(q^{3}\right)\right)^{2}}+\frac{\phi(q)-\phi\left(q^{3}\right)}{\phi(q)+\phi\left(q^{3}\right)} .
$$

On simplification of (68), we obtain

$$
\begin{aligned}
& \frac{1}{U^{2}(q)}+U(q)=\frac{2 \phi^{3}(q)+6 \phi(q) \phi^{2}\left(q^{3}\right)}{\left(\phi^{2}(q)-\phi^{2}\left(q^{3}\right)\right)\left(\phi(q)-\phi\left(q^{3}\right)\right)} \\
& \left(\frac{1}{U^{2}(q)}+U(q)\right)\left(\frac{\phi(q)}{\phi\left(q^{3}\right)}-1\right) \\
& =\frac{2 \phi(q)\left(\phi^{2}(q)+3 \phi^{2}\left(q^{3}\right)\right)}{\phi^{3}\left(q^{3}\right)\left(\frac{\phi^{2}(q)}{\phi^{2}\left(q^{3}\right)}-1\right)} .
\end{aligned}
$$

Employing (20), (26) and (30) in (69), we deduce that

$$
\frac{1+U^{3}(q)}{U(q)(1-U(q))}=\frac{\psi^{2}(q) \psi\left(q^{2}\right) \psi^{2}\left(q^{3}\right)}{q \phi^{2}\left(q^{3}\right) \psi^{3}\left(q^{6}\right)} .
$$

Using (22) in (70), we get (65).

By Lemma 2.1, we have

$$
\frac{1}{U^{3}(q)}+1=\frac{\left(\phi(q)+\phi\left(q^{3}\right)\right)^{3}}{\left(\phi(q)-\phi\left(q^{3}\right)\right)^{3}}+1 .
$$

On simplification of (71), we deduce

$$
\begin{aligned}
& \left(\frac{1}{U^{3}(q)}+1\right)\left(\frac{\phi(q)}{\phi\left(q^{3}\right)}-1\right)^{3} \\
& =2 \frac{\phi(q)\left(\phi^{2}(q)+3 \phi^{2}\left(q^{3}\right)\right)}{\phi^{3}\left(q^{3}\right)} .
\end{aligned}
$$


Employing (26) and (30) in (72) and after some simplifications, we get (66).

The proof of (67) is similar to the proof of (66).

Now, we shall establish identities involving $U(q)$, which are similar to the identities in Theorem 3.2 proved by B. Yuttanan [6].

Theorem 3.7. We have

$$
\begin{aligned}
& \frac{1}{\sqrt{U(q)}}+\alpha \sqrt{U(q)}=\frac{1}{q^{1 / 2}} \sqrt{\frac{\chi(-q) f\left(-q^{12}\right)}{\chi\left(-q^{3}\right)}} \\
& \left\{\prod_{n=1}^{\infty}\left(\begin{array}{l}
1+(\alpha-1)\left(\left(q^{1 / 3}\right)^{2 n}-\right. \\
\left.\left(q^{1 / 3}\right)^{n}\right)-q^{n}
\end{array}\right)-q^{1 / 3}\left(\frac{-\alpha+3}{-\alpha+4}\right)\right. \\
& \left.\times \prod_{n=0}^{\infty}\left(\begin{array}{l}
1-q^{12 n+3}\left(1+q^{9}+q^{6}-q^{12 n+9}\right. \\
\left.\left(1+q^{9}+q^{3}\right)+q^{24 n+21}\right)
\end{array}\right)\right\}
\end{aligned}
$$

and

$$
\begin{aligned}
& \frac{1}{\sqrt{U(q)}}+\beta \sqrt{U(q)}=\frac{1}{q^{1 / 2}} \sqrt{\frac{\chi(-q) f\left(-q^{12}\right)}{\chi\left(-q^{3}\right)}} \\
& \left\{\prod_{n=1}^{\infty}\left(1+(\beta-1)\left(\left(q^{1 / 3}\right)^{2 n}-\left(q^{1 / 3}\right)^{n}\right)-q^{n}\right)-q^{1 / 3}\left(\frac{\beta-3}{\beta-4}\right)\right. \\
& \left.\times \prod_{n=0}^{\infty}\left(\begin{array}{l}
1-q^{12 n+3}\left(1+q^{9}+q^{6}-q^{12 n+9}\right. \\
\left.\left(1+q^{9}+q^{3}\right)+q^{24 n+21}\right)
\end{array}\right)\right\},
\end{aligned}
$$

where $\alpha=2-\sqrt{3}$ and $\beta=2+\sqrt{3}$.

Proof. By the definition of $U(q)$, we have

$$
\frac{1}{\sqrt{U(q)}}+\alpha \sqrt{U(q)}=\frac{f\left(-q^{5},-q^{7}\right)+\alpha f\left(-q,-q^{11}\right)}{\sqrt{q f\left(-q,-q^{11}\right) f\left(-q^{5},-q^{7}\right)}} .
$$

Now, putting $k=6, a=\zeta$ and $b=\zeta^{11} q^{1 / 3}$ in (31), where $\zeta=e^{\pi i / 6}$, we obtain

$$
\begin{aligned}
& f\left(\zeta, \zeta^{11} q^{1 / 3}\right)=f\left(-q^{5},-q^{7}\right)+\zeta f\left(-q^{5},-q^{7}\right) \\
& +\zeta^{14} q^{1 / 3} f\left(-q^{3},-q^{9}\right)+\zeta^{39} q f\left(-q,-q^{11}\right) \\
& +\zeta^{76} q^{2} f\left(-q^{13},-q^{-1}\right)+\zeta^{125} q^{10 / 3} f\left(-q^{15},-q^{-3}\right) .
\end{aligned}
$$

Put $n=1, a=-q^{-1}$ and $b=-q^{13}$ in (32), to get

$$
f\left(-q^{-1},-q^{13}\right)=-q^{-1} f\left(-q,-q^{11}\right) .
$$

Again, setting $n=1, a=-q^{-3}$ and $b=-q^{15}$ in (32), we deduce

$$
f\left(-q^{-3},-q^{15}\right)=-q^{-3} f\left(-q^{3},-q^{9}\right) .
$$

Substituting (77) and (78) in (76), we get

$$
\begin{aligned}
& f\left(\zeta, \zeta^{11} q^{1 / 3}\right)=(1+\zeta) f\left(-q^{5},-q^{7}\right) \\
& +\left(\zeta^{39}-\zeta^{76}\right) q f\left(-q,-q^{11}\right) \\
& +\left(\zeta^{14}-\zeta^{125}\right) q^{1 / 3} f\left(-q^{3},-q^{9}\right) .
\end{aligned}
$$

Note that $\alpha=2-\zeta-\zeta^{11}$, so $\zeta^{39}-\zeta^{76}=\alpha(1+\zeta)$. It follows that

$$
\begin{aligned}
& \frac{f\left(\zeta, \zeta^{11} q^{1 / 3}\right)-\left(\zeta^{14}-\zeta^{125}\right) q^{1 / 3} f\left(-q^{3},-q^{9}\right)}{1+\zeta} \\
& =f\left(-q^{5},-q^{7}\right)+\alpha q f\left(-q,-q^{11}\right) .
\end{aligned}
$$

Now, using (80), Lemma 2.1 and Lemma 2.2 in (75), we obtain

$$
\begin{aligned}
& \frac{1}{\sqrt{U(q)}}+\alpha \sqrt{U(q)}=\frac{\sqrt{\chi\left(-q^{3}\right)}}{q^{1 / 2} \sqrt{\chi(-q) f^{2}\left(-q^{12}\right)}} \\
& \left\{\frac{f\left(\zeta, \zeta^{11} q^{1 / 3}\right)-\left(\zeta^{14}-\zeta^{125}\right) q^{1 / 3} f\left(-q^{3},-q^{9}\right)}{(1+\zeta)}\right\} .
\end{aligned}
$$

By Jacobi’s triple product identity,

$$
\begin{aligned}
& \frac{f\left(\zeta, \zeta^{11} q^{1 / 3}\right)}{(1+\zeta)}=\frac{\left(-\zeta ; q^{1 / 3}\right)_{\infty}\left(-\zeta^{11} q^{1 / 3} ; q^{1 / 3}\right)_{\infty}\left(q^{1 / 3} ; q^{1 / 3}\right)_{\infty}}{(1+\zeta)} \\
& =\left(-\zeta q^{1 / 3} ; q^{1 / 3}\right)_{\infty}\left(-\zeta^{11} q^{1 / 3} ; q^{1 / 3}\right)_{\infty}\left(q^{1 / 3} ; q^{1 / 3}\right)_{\infty} \\
& =\prod_{n=1}^{\infty}\left(1+\zeta\left(q^{1 / 3}\right)^{n}\right)\left(1+\zeta^{11}\left(q^{1 / 3}\right)^{n}\right)\left(1-q^{(1 / 3)^{n}}\right) .
\end{aligned}
$$

Since $1-\left(\zeta^{11}+\zeta\right)=1-\sqrt{3}$ and $\zeta^{12}=1$, we have

$$
\begin{aligned}
& \frac{f\left(\zeta, \zeta^{11} q^{1 / 3}\right)}{(1+\zeta)} \\
& =\prod_{n=1}^{\infty}\left(1+(1-\sqrt{3})\left(\left(q^{1 / 3}\right)^{2 n}-\left(q^{1 / 3}\right)^{n}\right)-q^{n}\right) \\
& =\prod_{n=1}^{\infty}\left(1+(\alpha-1)\left(\left(q^{1 / 3}\right)^{2 n}-\left(q^{1 / 3}\right)^{n}\right)-q^{n}\right) .
\end{aligned}
$$

Similarly, by Jacobi's triple product identity, we have

$$
\begin{aligned}
& \psi\left(-q^{3}\right)=\left(q^{3} ; q^{12}\right)_{\infty}\left(q^{9} ; q^{12}\right)_{\infty}\left(q^{12} ; q^{12}\right)_{\infty} \\
& =\prod_{n=0}^{\infty}\left(1-q^{12 n+3}\right)\left(1-q^{12 n+9}\right)\left(1-q^{12 n+12}\right) \\
& =\prod_{n=0}^{\infty}\left(1-q^{12 n+3}\left(1+q^{9}+q^{6}-q^{12 n+9}\right.\right. \\
& \left.\left.\left(1+q^{9}+q^{3}\right)+q^{24 n+21}\right)\right) .
\end{aligned}
$$

Note that

$$
\frac{\zeta^{14}-\zeta^{125}}{1+\zeta}=\frac{\sqrt{3}+1}{\sqrt{3}+2} .
$$

Substituting (82), (83) and (84) in (81), we get (73).

To prove (74), employ $\zeta^{51}-\zeta^{92}=\beta\left(1+\zeta^{5}\right)$ and $k=6, a=\zeta^{5}$ and $b=\zeta^{7} q^{1 / 3}$ in (31).

\section{Acknowledgements}

The first author is thankful to the University Grants Commission, Government of India for the financial support under the grant F.510/2/SAP-DRS/2011. The second author is thankful to UGC-BSR fellowship. The third author is thankful to DST, New Delhi for awarding INSPIRE Fellowship [No. DST/INSPIRE Fellowship/2012/122], under which this work has been done. 


\section{References}

[1] C. Adiga, B. C. Berndt, S. Bhargava and G. N. Watson, Chapter 16 of Ramanujan's second notebook: Theta functions and $q$-series, Mem. Amer. Math. Soc.,315 (1985), 1-91.

[2] C. Adiga, K. R. Vasuki and N. Bhaskar, Some new modular relations for the cubic functions, South East Asian Bull. Math.,36 (2012), 1-19.

[3] G. E. Andrews, On q- difference equations for certain well-poised basic hyoergeometric series, Quart. J. Math. (Oxford), 19 (1968), 433-447.

[4] N. D. Baruah and R. Barman, Certain theta function identities and Ramanujan's modular equations of degree 3, Indian J. Math.,48 (3) (2006), 113-133.

[5] B. C. Berndt, Ramanujan's Notebooks, Part III, Springer-Verlag, New York, 1991.

[6] S. Bhargava, C. Adiga and D. D. Somashekara, Ramanujan's remarkable summation formula and an interesting convolution identity, Bull. Austral. Math. Soc., 47 (1993), 155-162.

[7] Boonrod Yuttanan, New properties for the Ramanujan-GöllnitzGordon continued fraction, Acta Arithmetric, 151(3) (2012), 293-310.
[8] H. Göllnitz, Partitionen mit Diffrenzenbedinguggen, J. Reine Angew Math, 225 (1967), 154-190.

[9] B. Gordon, Some continued fractions of the Rogers-Ramanujan type, Duke Math. J. 32 (1965), 741-748.

[10] M. S. Mahadeva Naika, B. N. Dharmendra and K. Shivashankar, A continued fraction of order twelve, Centr. Eur. J. Math., 6 (3) (2008), 393-404.

[11] S. Ramanujan, Notebooks (2 volumes), Tata Inst. Fund. Res., Bombay, 1957.

[12] H. M. Srivastava, Some convolution identities based upon Ramanujan's bilateral sum, Bull. Austral. Math. Soc., 49 (1994), 433-437.

[13] K. R. Vasuki, Abdulrawf A. Kahtan, G. Sharth and C. Sathish Kumar, On a continued fraction of order 12, Ukra. Math. J., 62 (12) (2010), 1866-1878.

[14] K. R. Vasuki, G. Sharth and K. R. Rajanna, Two modular equations for squares of the cubic functions with applications, Note di Math.30 (2) (2010), 61-70.

[15] K. W. Yang, On the product $\prod_{n \geq 1}\left(1+q^{n} x+q^{2 n} x^{2}\right)$, J.Austral. Math. Soc., Ser.A 48 (1990), 148-151. 